\title{
Detecção de áreas de clareira na Floresta Amazônica através de monitoramento via satélite e aprendizagem de máquina
}

\author{
Marcela Pessoa $^{1,2}$, Robson Melo ${ }^{1}$, Adrisson Rodrigues ${ }^{4}$, \\ Sergio Cleger ${ }^{3}$, João Marcos Cavalcanti ${ }^{1}$, Rosiane de Freitas ${ }^{1}$ \\ ${ }^{1}$ PPGI/Instituto de Computação - Universidade Federal do Amazonas \\ ${ }^{2}$ Escola Superior de Tecnologia - Universidade do Estado do Amazonas \\ ${ }^{3}$ SIDIA Instituto de Ciência e Tecnologia \\ ${ }^{4}$ Faculdade Fucapi \\ Manaus - AM - Brazil \\ \{rosiane, john\}@icomp.ufam.edu.br, s.tamayo@sidia.com
}

\begin{abstract}
This paper addresses the problem of clearing detection in the Amazon rainforest using remote sensing data and (un)supervised machine learning techniques. A clearing (or canopy gap) is a small area in a forest where there are no trees, or there is only low-lying forest which differs from its surroundings. Experiments carried out with 44 satellite images, divided into 3.288 segments, evaluated by experts, where 1, 652 of non-clearing class and 1, 636 of clearing. The segments were represented by a set of features that include first and second order statistics and color information. In the supervised learning approach, the best results were obtained with the rule-based methods, Decision Tree and Random Forest, reaching $97 \%$ in both classes. Methods that use different approaches have got worse results, which suggests the need for a better analysis of the relation between the attributes. Among the unsupervised learning techniques, the best performance was achieved by the BIRCH method, which obtained $94.48 \%$ for both classes. However it required a higher number of clusters under higher execution time. Preliminary results indicate that the proposed approach is promising, which must continue to be investigated.
\end{abstract}

Resumo. Este artigo aborda o problema de detecção de áreas de clareira na Floresta Amazônica, através do uso de dados de sensoriamento remoto e técnicas de aprendizagem de máquina supervisionada e não-supervisionada. Uma clareira (ou lacuna de dossel) é uma pequena área em uma floresta onde não há árvores ou há apenas mata baixa que destoa do seu entorno. Foram usadas 44 imagens de satélite, divididas em 3.288 segmentos, rotulados por um especialista, sendo 1.652 da classe não-clareira e 1.636 de clareira. Os segmentos foram representados por um conjunto de características que inclui informações de cor e estatística de $1^{a}$ e $2^{a}$ ordem. No aprendizado supervisionado, os melhores resultados foram obtidos com os métodos baseados em regras, Decision Tree e Randon Forest, chegando a $97 \%$ nas duas classes. Métodos que usam abordagens diferentes obtiveram resultados piores, o que sugere melhor análise da relação entre os atributos. Já no aprendizado não-supervisionado, o melhor desempenho foi para o método BIRCH, que obteve $94,48 \%$ para as duas classes, mas necessitando de um grande número de agrupamentos sob alto custo de processamento. Os resultados preliminares obtidos indicam que a abordagem proposta é promissora, o que deve continuar sendo investigado. 


\section{Introdução}

O bioma Amazônia é uma enorme região, que cobre parte de nove países da América do Sul, sendo que 69\% dessa região pertence ao Brasil [MapBiomas 2015] [Ab'Sáber 1997], totalizando uma área de $4.871 .000 \mathrm{~km}^{2}$ a ser dividida entre os estados brasileiros do Amazonas, Pará, Maranhão, Tocantins, Mato Grosso, Acre, Amapá, Rondônia e Roraima. Ao longo dos anos, tais áreas de floresta vêm sendo devastadas, seja por atividade madeireira ilegal, por invasão de terras para criação de pastos, ou ainda pela atividade ilegal de garimpeiros, causando um aumento do desmatamento. A consequência desse desmatamento pode vir de diferentes formas, tais como, diminuição da biodiversidade, desequilíbrio ecossistêmico e altas concentrações de carbono atmosférico que prejudicam o equilíbrio do clima, dentre outras consequências.

O governo brasileiro utiliza o Sistema de Monitoramento do Desflorestamento da Floresta Amazônica (PRODES) para acompanhar o desmatamento deste Bioma a partir da interpretação de imagens de sensoriamento remoto. Porém, ainda não se conseguiu que processos totalmente automatizados de segmentação e classificação gerem resultados, em uma escala aceitável de satisfação, na detecção de clareiras (ou lacunas de dossel - copa de árvores), que são justamente áreas de pequena dimensão em uma floresta onde não há árvores ou há apenas mata baixa que destoa do seu entorno, podendo ser fruto de ação do homem (elementos antrópicos) ou de fenômenos naturais, como o efeito Blowdown [Magnabosco et al. 2014], que gera lacunas de dossel (clareiras) criadas por eventos de arremesso de vento, ou blowdowns, criando um complexo mosaico de fragmentos florestais na Amazônia Central.

Se por um lado existe uma vasta área imageada por sensoriamento remoto, oriundas de várias tecnologias ()como Satélite, Radar e LIDAR) e com melhoria crescente na qualidade das imagens, por outro, ainda se carece de procedimentos para analisar essa grande quantidade de dados e propor soluções computacionais a partir disto. Por esta razão, este tema tem despertado o interesse de pesquisadores na área da computação, especialmente em como identificar, de forma automatizada, áreas de clareira e quais seriam as melhores técnicas de segmentação e classificação de imagens.

Pesquisas nesta área envolvem diversas etapas, tais como: i) elaborar ou determinar a técnica e tecnologias de sensoriamento remoto mais adequada para captura das imagens; ii) determinar como tais imagens serão consideradas (p.ex., divididas pixel a pixel ou regiões); iii) determinar como interfacear com um especialista que possa rotular os dados antes ou validar depois; iv) elaborar modelos e técnicas de segmentação e/ou classificação que possam gerar melhores resultados, v) determinar qual conjunto de características pode ser usado para descrever as imagens. Na abordagem por aprendizagem de máquina todas essas etapas são importantes para compor a base de dados e se determinar quais técnicas de classificação podem ser usadas para aumentar a acurácia das predições.

Dado este contexto, neste trabalho é apresentada uma análise comparativa entre algoritmos de aprendizagem de máquina supervisionada e não-supervisionada, como forma de buscar uma técnica automatizada de detecção de clareiras em regiões de floresta Amazônica. Imagens obtidas por satélite e um conjunto de características de cor e textura foram utilizadas como base dos métodos de classificação. Experimentos utilizando diversas técnicas de classificação foram realizados, incluindo abordagens supervisionadas e não-supervisionadas, para avaliar qual técnica apresenta melhores resultados com base 
nas características das imagens fornecidas.

Aprendizagem de Máquina é a parte da Inteligência Artificial cujo objetivo é encontrar e descrever padrões estruturais em dados como uma ferramenta para ajudar a explicá-los e fazer predições a partir deles [Witten and Frank 2005]. Basicamente, a aprendizagem de máquina pode ser de três tipos: i) Supervisionada; ii) Não Supervisionada e iii) Aprendizagem por Reforço. Neste trabalho estão sendo usadas técnicas supervisionadas e não-supervisionadas. Aprendizagem Supervisionada é um conjunto de observações em que a classe de cada exemplo é conhecida. O objetivo é encontrar uma hipótese capaz de classificar novas observações entre as classes já existentes [Russell and Norvig 2003]. Entre as tarefas de Aprendizagem Supervisionada estão a Classificação e a Regressão. Já em Aprendizagem Não-supervisionada, dado um conjunto de observações ou exemplos sem rótulos, o objetivo é tentar estabelecer a existência de grupos ou similaridades nesses exemplos [Russell and Norvig 2003]. Esses grupos de objetos são denominados cluster. Um cluster é uma coleção de dados de objetos que são similares uns aos outros. Ao contrário da aprendizagem supervisionada que tem o custo de rotular as classes, na nãosupervisionada isto não é necessário uma vez que os algoritmos vão agrupar as instâncias pela similaridade que há entre elas.

Sobre Sensoriamento Remoto (SR), denomina-se um conjunto de técnicas destinado à obtenção de informação sobre objetos, sem que haja um contato físico com eles [Curran 1988]. No sensoriamento remoto são usados comprimentos de onda que abrangem o ultravioleta, todo o espectro visível (observado pelos olhos humanos), partes do infravermelho e microondas. O espectro visível é dividido basicamente em sete faixas das quais as principais são azul, verde e vermelho. Entre os tipos de tecnologias existentes pode-se citar o Radar e o Satélite. Uma vantagem do satélite em relação ao Radar é que, pelo fato de atuar no espectro visível, permite transformar três de cada uma das sete faixas ou bandas em efeitos significativos e, com isso trabalhar, com imagens coloridas, usando tons de cores bem próximos do que o olho humano enxerga. Para este trabalho foi escolhido trabalhar com imagens de Satélite para poder tirar proveito das características relacionadas a cor.

O restante deste artigo está organizado como segue: na Seção 2 são apresentados alguns trabalhos relacionados; na Seção 3 é descrita a proposta metodológica adotada; na Seção 4 são apresentados os experimentos computacionais e a análise dos resultados; por fim, na Seção 5 são feitas as considerações finais.

\section{Trabalhos Relacionados}

Em trabalhos cujo foco é explorar informações presentes numa imagem, uma das tarefas mais importantes é definir um conjunto de características que melhor descreva a imagem ou um segmento dela. Neste contexto, algumas das características que chamam a atenção de pesquisadores estão relacionadas à textura da imagem. Textura é uma representação efetiva da informação contextual e espacial. Várias medidas de texturas baseadas em estatísticas de histograma, matriz de co-ocorrência de nível de cinza (GLCM) e campos aleatórios de Markov (MRFs) foram amplamente investigados na classificação de imagens de satélite [Dell'Acqua and Gamba 2003][He et al. 2013][Uhlmann and Kiranyaz 2014].

Uma área de crescente interesse entre os pesquisadores está relacionada a imagens registradas através de sensoriamento remoto. Várias trabalhos tentam explorar 
informações oriundas de tecnologias como Radar, Satélite e LiDAR. Este crescente número de dados gerados por tecnologias de Sensoriamento Remoto também despertou o interesse na comunidade de aprendizagem de máquina, especialmente para a criação de modelos de classificação automática. Na atividade de classificação o melhor desempenho não depende apenas da robustez dos classificadores mas também da qualidade das características de entrada e do tamanho da base de treinamento [Olofsson et al. 2014]. Tal como é importante a escolha do classificador e o tamanho da base, a extração das características e exemplos tem atraído a atenção de pesquisadores, a partir de diferentes aspectos na classificação de imagem [Qi et al. 2012] [He et al. 2013] [Jin et al. 2014].

Pla [Pla 2013] descreve o estado da arte de classificação unária para reconhecimento de imagens e mostra uma aplicação de classificação de pixels. Usam base de imagens hiperespectrais de satélite, com 1232 instâncias, contendo duas classes que são vegetação, com maior número de instâncias, e solo, como classe minoritária. Os autores apresentam taxas de acertos de $100 \%$ para a classe vegetação e somente $9 \%$ foram classificadas como vegetação e sendo da classe solo. Não fica claro como se dá distribuição das classes e nem apresenta resultados sobre a classe minoritária. Já Wang [Wang 2013] compara métodos de detecção de anomalias utilizando classificadores unários, para detectar elementos anômalos em imagens hiperespectrais de satélite. São utilizadas duas bases de dados de imagens, uma de regiões florestais e agrárias do estado de Indiana e a outra de imagens urbanas de San Diego, nos Estados Unidos. Buscando melhores resultados, eles mesclam características espaciais e espectrais. O melhor resultado foi de $94 \%$ no SCSVDD (Spatial-Contextual Support Vector Data Description) que é uma variação do SVM voltado para o problema de classificação unária e foi bem adaptado ao problema apresentado. Kumar e Miller [Kumar and Miller 2006] propõem um modelo de classificação de objetos na imagem usando informação de cor e de textura. A textura é usada nos casos onde a cor da área não é suficiente para caracterizar a classe. A classificação é supervisionada pixel a pixel de um total de 16 áreas de cobertura, incluindo a zona urbana, com classificador máxima verossimilhança. Os resultados mostram que as classes rotuladas usando somente informação de cor atingiram um máximo de $80 \%$ dos pixels de classe, enquanto que as classes que adicionaram a informação de textura combinada à cor, conseguiram atingir 100\% de classificação dos pixels em algumas classes.

Enquanto que o trabalho de Braga e Figueiredo [Braga and Figueiredo 2006] compara os processos de classificação supervisionada e não-supervisionada no mapeamento do uso do solo. A classificação supervisionada apresenta melhores resultados se comparada à não-supervisionada, atribuem o resultado ao fato de que no primeiro o analista tem maior controle na classificação, possibilitando a identificação de imprecisões nas áreas de treinamento antes da classificação. No entanto, eles destacam o uso da classificação não-supervisionada quando o usuário não conhece bem a área a ser classificada, e principalmente se esta envolve uma estratificação temática mais detalhada (multi-classes). Em Du [Du et al. 2015] é proposto um modelo de classificação de imagens que utiliza os métodos Random Forest e Rotation Forest. Eles propõem a combinação de características polarimétrica e espacial. Usaram três bases de imagem. A primeira de 600x1200 pixels, da região de San Francisco Golden Gate Bridge, organizadas em cinco classes, incluindo região de água, vegetação e construção. A segunda base tem 1400x1000 pixels da região de Felvoland e contém cinco classes, entre elas região de água e floresta. No terceiro conjunto foi usada imagem da região de Netherlands, medindo 1300x1500 pixels, com 
4 classes também tendo entre elas região de floresta e água. Embora o Rotation Forest supere o Random Forest em termos de acurácia nos três experimentos executados, até mesmo na base menor, sua eficiência é prejudicada porque é altamente afetado pela dimensionalidade das características, sendo até dez vezes mais rápido do que o Rotation Forest. Outro ponto a se destacar é que na classe região de água houve acurácia de até $100 \%$ para alguns algoritmos.

$\mathrm{Na}$ literatura são encontrados vários trabalhos relacionados à segmentação de imagem e à avaliação de características que descrevem pixel ou segmento de imagem [Olofsson et al. 2014] [Qi et al. 2012] [He et al. 2013] [Jin et al. 2014] [Dell' Acqua and Gamba 2003] [Uhlmann and Kiranyaz 2014] porém ainda são escassos os trabalhos que estudam imagens de florestas, em especial Floresta Amazônica, neste contexto, Cavalcanti et al. [Cavalcanti L. et al. 2015] compara vários algoritmos comuns de classificação e avalia sua confiabilidade na segmentação de imagens aéreas de regiões de floresta tropical. Para os experimentos eles usam imagens reais de um conjunto de dados disponível publicamente. Com mais de $90 \%$ de precisão, alguns dos algoritmos de aprendizado de máquina testados são boas abordagens para classificação de imagens de florestas aéreas com baixa resolução. O Random Forest apresentou um desempenho melhor no conjunto de dados, mas os autores destacam a necessidade de resolver o problema de falso positivos em segmentos de imagem que representam água, que, segundo eles, é visivelmente maior do que em outros métodos. Aqui cabe comparar com Du [Du et al. 2015] que, os melhores resultados foram ao classificar classes com regiões de água, enquanto para Cavalcanti et al. [Cavalcanti L. et al. 2015] teve seu pior resultado. Este trabalho é uma extensão do proposto por Cavalcanti et al. [Cavalcanti L. et al. 2015].

\section{Metodologia Proposta}

Para o desenvolvimento deste trabalho foi seguida a metodologia apresentada na Figura 1, que está dividida em três etapas: Extração das Características, Aprendizagem e Validação. Tais etapas são descritas não subseções a seguir.

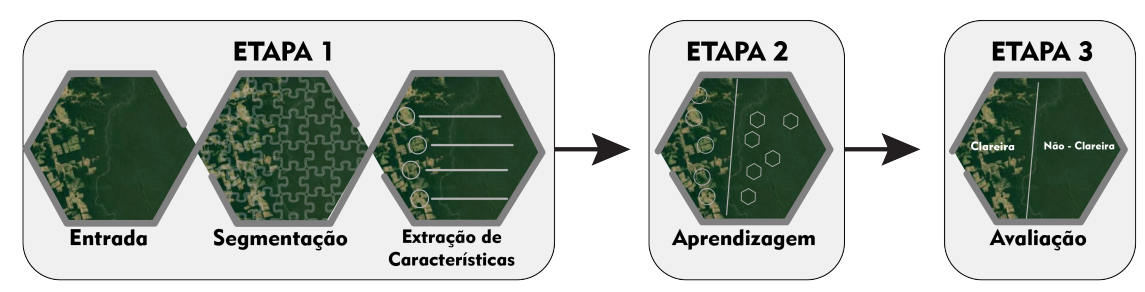

Figura 1. Metodologia utilizada na pesquisa, apresentando as três etapas principais: extração das características, aprendizagem e avaliação.

Neste trabalho estão sendo usados algoritmos SVM, Decision Tree, Perceptron, Random Forest, Logistic Regression, Nearest Neighbour Algorithm (KNN) e Gaussian Naive Bayes.

A classes são clareira (Desmatamento) ou não-clareira (Não-Desmatamento), definidos por valores discretos 0 e 1 . Para o aprendizado não-supervisionado, Foram utilizados três algoritmos: i) Algoritmos K-Means, que agrupa os dados tentando dividir as amostras em $n$ grupos de igual variância, minimizando a soma dos quadrados dentro 
de cada grupo [Tan et al. 2009]; ii) Agrupamento Hierárquico Aglomerativo (Agglomerative Clustering), é um conjunto de técnicas de agrupamento relacionadas que produz um agrupamento hierárquico iniciando com cada ponto como um grupo único e depois fundindo repetidamente os dois grupos mais próximos até que reste um único grupo [Tan et al. 2009], e iii) BIRCH (Balanced Iterative Reducing and Clustering using Hierarchies) que constrói uma estrutura de dados em árvore com os centróides do cluster sendo lidos da folha. Estes podem ser os centróides do cluster final ou podem ser fornecidos como entrada para outro algoritmo de clustering, como o Agglomerative Clustering.

Neste trabalho foi necessário medir o desempenho do modelo no conjunto de teste para fazer uma avaliação imparcial do erro de generalização. foi utilizado o método de Validação Cruzada (Cross Validation), com 10 partições, k-folds, que consiste em dividir o conjunto de dados em $k$ partes. Durante cada execução uma das partições é escolhida para teste, enquanto que as outras são usadas para treino. Este procedimento é repetido $k$ vezes de modo que cada partição seja usada para teste pelo menos uma vez. O erro total é encontrado pela soma dos erros de todas as $k$ execuções [Tan et al. 2009] e o método Leave one out é retirada uma instância para teste e as demais são usadas para treino,repetindo em todo o conjunto de dados.

\subsection{Etapa 1: Extração das Características}

Esta etapa é responsável por pegar um conjunto de 44 imagens de satélite, segmentá-las e extrair características que sejam capazes de melhor representar a imagem, conforme seguintes fases:

\subsubsection{Entrada e Segmentação}

A entrada é composta por uma base de imagens de satélite com $30 \mathrm{~m}^{2}$ de resolução espacial correspondente ao sensor óptico Landsat TM-5. As imagens foram cedidas pelo SIPAM (Sistema de Proteção da Amazônia) e cobre a área de desmatamento do município de Barcelos, no Amazonas.

No processo de segmentação, a imagem foi dividida em regiões, rotuladas por especialista na área, divididas em duas classes: clareira e não-clareira. Após a segmentação se obtém um conjunto de regiões representativas de cada uma das classes analisadas. Como as imagens têm baixa resolução, foram considerados, no mínimo 700 pixels para formar um segmento. A base, formada por 26 imagens, é dividida em 3.288 segmentos, sendo 1.652 segmentos de área de não-clareira e 1.636 segmentos de área de clareira.

\subsubsection{Seleção de Características}

As características usadas neste trabalho foram definidas por Fernandes [Fernandes 2008], e envolve o histograma de cor, preenchendo o segmento da posição 1 à posição 256 para a banda R, de 257 a 512 para a banda $\mathrm{G}$, de 513 a 768 para a banda B. Também são adicionadas informações de medida de textura, ou seja, as principais medidas da estatística de primeira ordem como média, variância e desvio padrão de cor para as bandas RGB (VR, VG, VB), média de cor para as bandas RGB (MR, MG, MB) e desvio padrão para as bandas RGB (DPR, DPG, DPB) . No total foram definidas 781 características. Para evitar 
que valores altos influenciassem no comportamento dos algoritmos, os dados foram normalizados para uma escala logarítmica que reduz em 28 vezes a distância entre o menor e o maior valor possível em cada posição do histograma, ou seja, os valores originalmente entre 0 e 255 são normalizados para valores entre 0 e 9.

Não foi usada informação de forma para caracterizar clareira já que a imagem foi dividida em regiões de formato irregular, dificultando trabalhar com o formato geométrico, que caracteriza as feições de desmatamento neste tipo de tratamento de imagem. Inclusive, em alguns casos, dependendo da imagem, terão regiões mal formadas ou incompletas, fazendo com que a caracterização de forma do segmento fique irrelevante.

\subsection{Etapa 2: Processo de Aprendizagem}

Na etapa de Aprendizagem é realizado o Treinamento e a Classificação. Para o Treinamento, após os segmentos serem representados pelas 781 características, estas são convertidas em vetores que são utilizados como entrada para o processo de treinamento dos classificadores. Com base nessas características e considerando que regiões parecidas tem características parecidas, acredita-se que o classificador seja capaz de aprender as características de cada região similar. Como resultado do processo, um modelo de classificação é obtido e este deve ser capaz de predizer a classe de novos segmentos de imagens apresentados ao classificador, para posteriormente ser validado na fase de validação.

Foram utilizadas as abordagens de classificação supervisionada e nãosupervisionada, utilizando a estratégia de classificação binária, considerando as classes clareira e não-clareira. Para a aprendizagem supervisionada foram utilizados os algoritmos SVM, Decision Tree, Perceptron, Random Forest, Logistic Regression, Nearest Neighbour Algorithm (KNN) e Gaussian Naive Bayes com validação cruzada $k$-Fold, leave one out. Para a aprendizagem não-supervisionada foram utilizados os algoritmos: K-means, Agglomerative Clustering e BIRCH.

Os parâmetros utilizados nos algoritmo de aprendizagem foram decididos na fase de treinamento. Foram testados vários parâmetros em cada método mas para registro neste artigo foram considerados os melhores resultados. Não foram introduzidos parâmetros nos métodos: SVM, Decision Tree, Logistic Regression e GNB. Entre os parâmetros usados para o classificador KNN, foi variado o número de vizinhos em um intervalo de $1 \mathrm{a}$ 5, sendo o melhor resultado com k=5 e distância euclidiana. No classificador Random Forest o maior número de árvores foi 1000 e sem limitações do uso das características. Para o perceptron foram usadas 3.000 entradas com uma taxa de aprendizagem de 0.2. Já o método SGD foi utilizado limitando o número máximo de iterações a 500.

\subsection{Etapa 3: Validação}

Para validar o modelo criado no treinamento, foi utilizado um conjunto de segmentos de imagens de teste, excluindo a classe. O modelo classifica as instâncias e compara o resultado com a classe real segmento. Desta forma, é possível verificar quantas instâncias o classificador classificou corretamente e avaliar a precisão de cada um dos classificadores.

O conjunto de teste é independente do conjunto de treino, ou seja, a base de teste possui segmentos de imagens diferentes do conjunto utilizado para o treino. 


\section{Experimentos e Resultados}

Com o objetivo de detectar áreas de clareira em região de floresta tropical, foram considerados três aspectos para os experimentos: 1) Aprendizagem Supervisionada com validação cruzada (crosss validation), 2) Aprendizagem Supervisionada com particionamento dos dados através da técnica leave one out e 3) Aprendizagem não-supervisionada.

\subsection{Experimento 1: Aprendizagem Supervisionada com Validação Cruzada}

Na Tabela 1 são apresentadas as médias da acurácia das classificações para as instâncias usando os sete classificadores. Foi utilizado o teste estatístico de Friedman, para verificar se existem diferenças entre os resultados de pares de modelos ( $\operatorname{com} \mathrm{p}<0,05)$.

Os resultados sugerem que a distribuição da dados é aleatória, fazendo com que modelos como SVM, Perceptron e Logistic Regression não consigam encontrar o melhor hiperplano para classificar com maior precisão. Em geral o melhor resultado é obtido com Decision Tree (com taxa de 97,18\% para a classe clareira e 97,65\% para a classe não-clareira), apresentando significativas diferenças em relação aos modelos restantes . $\mathrm{O}$ Randon Forest também apresenta melhores resultados em relação aos demais modelos.

Os melhores modelos na classificação estão relacionados à utilização de árvores (Decision Tree e Randon Forest) e, portanto, na obtenção de regras. Isto sugere que sejam estudadas ramificações e relações entre os atributos, o que será feito em pesquisas futuras.

Tabela 1. Resultado do experimento com cross validation

\begin{tabular}{r|l|r|l}
$\#$ & Método & Clar \% & NClar \% \\
\hline 1 & SVM & 43,15 & 74,15 \\
\hline 2 & DecTree & $\mathbf{9 7 , 1 8}$ & $\mathbf{9 7 , 6 5}$ \\
\hline 3 & Percep & 68,94 & 56,53 \\
\hline 4 & RandFor & 87,12 & 84,79 \\
\hline 5 & LogReg & 83,20 & 74,65 \\
\hline 6 & KNN & 78,77 & 77,09 \\
\hline 7 & NB & 64,02 & 82,85
\end{tabular}

Tabela 2. Resultado do experimento com Leave One Out

\begin{tabular}{r|l|r|l}
$\#$ & Método & Clar \% & NClar \% \\
\hline 1 & SVM & 44,75 & 74,13 \\
\hline 2 & DecTree & $\mathbf{1 0 0}$ & $\mathbf{1 0 0}$ \\
\hline 3 & Percep & 84,19 & 26,54 \\
\hline 4 & RandFor & 87,75 & 85,06 \\
\hline 5 & LogReg & 85,20 & 75,17 \\
\hline 6 & KNN & 83,38 & 79,41 \\
\hline 7 & NB & 64,20 & 83,12
\end{tabular}

\subsection{Experimento 2: Aprendizagem Supervisionada com Leave One Out}

Quanto à utilização de mais valores no conjunto de treino (leave one out), os resultados foram similares aos obtidos com validação cruzada de 10 folds em relação às características. Os métodos baseados em regras mostram os resultados mais estáveis e reafirmam a hipótese de realizar um estudo mais aprofundado para verificar a correlação entre as características disponíveis. A Tabela 2 apresenta o resultado dos experimentos usando a técnica Leave One Out, aplicada aos sete métodos de aprendizagem de máquina supervionada experimentados.

Os resultados obtidos com o algoritmo Decision Tree, apontam para a necessidade de testar os modelos obtidos com outros trechos de floresta tropical e assim avaliar a possibilidade de generalização, além de validar a hipótese de que são necessários dados representativos em maior quantidade e qualidade. 


\subsection{Experimento 3: Classificação Não-supervisionada}

Foram utilizados três métodos de classificação não-supervisionada: K-means, Agglomerative Clustering e BIRCH. Nestes algoritmos, a classificação de novas instâncias é feita pelos grupos formados, onde se utiliza como critério a maioria de instâncias de uma determinada classe para classificar. Na Tabela 3 são apresentadas as médias de acurácia dos métodos não-supervisionados avaliados, para os diferentes números de grupo formados.

Tabela 3. Médias de acurácia dos métodos não-supervisionados experimentados neste trabalho

\begin{tabular}{c|c|c|c|c|c|c} 
& \multicolumn{2}{|c|}{ K-means } & \multicolumn{2}{c|}{ AC } & \multicolumn{2}{c}{ BIRCH } \\
\hline \# de Clusters & Clar \% & NClar \% & Clar \% & NClar \% & Clar \% & NClar \% \\
\hline 400 & 52,75 & 52,60 & 22,00 & 47,30 & 58,20 & 51,60 \\
\hline 700 & 60,50 & 59,71 & 28,00 & 51,60 & 63,70 & 61,60 \\
\hline 1000 & 63,00 & 67,00 & N/A & N/A & 73,50 & 72,00 \\
\hline 1300 & 74,75 & 80,00 & N/A & N/A & 85,00 & 85,30 \\
\hline 1500 & 85,00 & 86,90 & N/A & N/A & 91,50 & 92,40 \\
\hline 1600 & 88,75 & 90,00 & N/A & N/A & 94,48 & 94,48 \\
\hline 1700 & 91,25 & 92,78 & N/A & N/A & 94,48 & 94,48 \\
\hline 1800 & 94,78 & 95,66 & N/A & N/A & 94,48 & 94,48
\end{tabular}

No modelo BIRCH os resultados melhoraram com o aumento do número de clusters, não apresentando diferença nos resultados a partir de 1.545 clusters. Esta técnica obteve acurácia de $94,48 \%$, representando o melhor comportamento quando comparada com as outras duas técnicas não-supervisionadas em relação ao número de clusters predição. O principal problema está no elevado número de clusters necessários para a melhor predição, no mínimo 1.300, para obter valores superiores a 86\%, isso implica em custo computacional elevado. No caso deste algoritmo, as melhores classificações são obtidas com grupos de no máximo 2 instâncias. A relação obtida nesta experimentação entre o número de instâncias e a acurácia, sugere a necessidade de avaliar a utilização destes métodos para conjuntos de dados com um número maior de instâncias.

No caso do K-means o incremento no número de clusters também é um fator que incide na predição. Para obter valores superiores a $90 \%$ é preciso aumentar até 1700 clusters. No caso de $K$-means é necessário chegar aos 1800 grupos para melhorar os resultados do método BIRCH. Neste método o número de instâncias em cada grupo formado pode variar entre 1 e 4 instâncias.

No Agglomerative Clustering, foi possível utilizar até 822 clusters, sendo em todos os casos resultados menores que $63 \%$ e significativamente inferiores quando comparado com os outros dois métodos avaliados. Como é possível observar, neste algoritmo foi formado um número menor de clusters, o que incide diretamente no número de instâncias em cada cluster, chegando até a 22 instâncias.

Nas técnicas não-supervisionadas usadas, o principal problema está na definição do número de clusters, que pode ser diferente para outros conjuntos de dados. Porém, este tipo de abordagem se mostra como uma possível solução para problemas com instâncias não-classificadas (problema comum na área de sensoriamento remoto). Em relação ao tempo de processamento/geração dos modelos, em todos os casos testados foram menores que dois segundos, fato considerado positivo na utilização destes algoritmos. 


\subsection{Análise Comparativa dos Resultados}

É evidenciado neste trabalho que as abordagens de aprendizagem de máquina supervisionada e não-supervisionada se mostram relevantes para o problema de detecção de pequenas áreas de mata baixa ou clareiras. Os resultados sobre a base de satélite analisada se mostram promissores em relação a outras pesquisas referenciadas neste trabalho. Em relação ao número de atributos, os valores de precisão indicam a necessidade de realizar experimentos adicionais para avaliar a relevância e influência (dependência e independência) das características sob resultados. Os classificadores baseados em regras, como Decision Tree e Random Forest, que apresentam resultados significativos em todos os experimentos quando comparados aos restantes, corroboram com esta hipóteses.

Um dos grandes problemas em aplicações que utilizam dados oriundos de sensoriamento remoto é a falta de bases de dados classificadas, especialmente em florestas tropicais. Neste cenário, as técnicas de aprendizagem de máquina não-supervisionadas se apresentam como soluções viáveis, porém, com um custo computacional associado à formação do número de grupos. Neste trabalho o tempo de processamento não foi um impeditivo para a geração e verificação dos modelos, entretanto, deve ser um elemento a ser considerado em trabalhos futuros, especialmente os que utilizarem bases de dados maiores e multi-classes. Fatores como o número de instâncias por grupos poderiam ser utilizados como limiar de confiança em base de dados maiores.

Apesar da base de dados utilizada neste trabalho estar balanceada, esta não é uma característica comum a um conjunto de dados que reflete a realidade deste problema, o que sugere a necessidade de validação dos modelos obtidos em outros trechos de florestas tropical. Já o tempo de processamento nas duas abordagens não teve diferenças significativas, fato associado ao baixo número de instâncias. Em relação a este quesito, não é possível chegar a uma conclusão, indicando que sejam realizados novos estudos, com bases de dados maiores em busca de analisar seu comportamento.

A Figura 2 a seguir apresenta duas imagens. A primeira delas (a) é uma imagem de satéliste LANDSAT de uma região ao Sul do estado do Amazonas, rotulada por especialista. A segunda (b) é a imagem após segmentação e aplicação de técnicas para detecção de clareiras, onde pode ser observado que foram eficientes na detecção das áreas rotuladas pelo especialista.
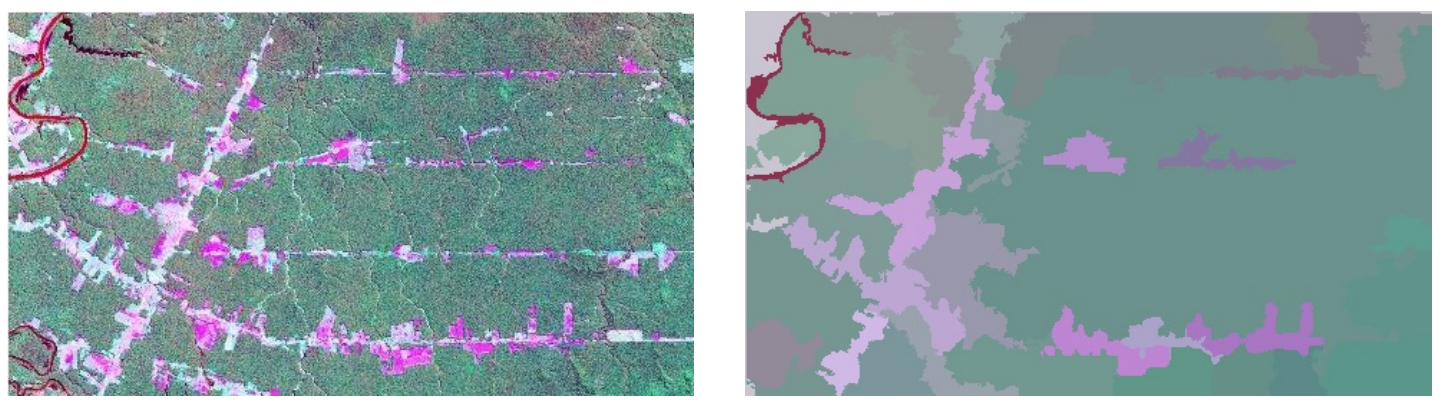

Figura 2. (a) Imagem original classificada de Satélite LANDSAT. (b) Imagem segmentada com áreas de clareira detectadas. 


\section{Considerações Finais}

A metodologia proposta neste trabalho para classificar segmentos de floresta como clareira ou não-clareira, de forma automatizada, com base na definição de características baseadas em cor e textura que representam um segmento, se mostrou adequada de modo a serem detectadas as áreas rotuladas por um especialista, com um grande percentual de acerto. Foram usadas 44 de imagens de satélite, dividida em 3.288 segmentos. Cada segmento foi representado um conjunto de 781 características, oriundas de informações de cor e também de estatísticas de primeira e segunda ordem, como média, variância e desvio padrão.

Para fazer a previsão das classes foram utilizados algoritmos de aprendizagem de máquina, tanto supervisionada como não-supervisionada. Para os algoritmos supervisionados foram utilizados os métodos de partição e validação cruzada. Os resultados mostram que o algoritmo Decision Tree teve melhor desempenho, despertando o interesse em investigar uma possível correlação entre as características. Já nos algoritmos não-supervisionados, os resultados foram melhores no método $\mathrm{BIRCH}$, que teve como desvantagem precisar de um número muito grande de agrupamentos (clusters).

Grande parte da Floresta Amazônica é coberta por sistemas de sensoriamento remoto de alta resolução. O maior problema para utilizar esses dados é a falta de classificação das imagens ou segmentos de imagens, para que seja possível utilizar aprendizagem supervisionada. Dado este cenário, como possíveis extensões e trabalhos futuros, pode-se: i) desenvolver métodos que utilizem aprendizado ativo para diminuir o número de dados classificados; ii) utilizar dados de sensoriamento remoto de fontes heterogêneas de uma mesma área, por exemplo de Radar e LiDAR, para avaliar o impacto das características, refinar o modelo e obter maior acurácia na detecção das áreas desejadas; iii) melhorar o algoritmo de segmentação de imagens; iv) preparar uma base de maior escala (ou construir outras bases de dados da floresta amazônica) para utilizar também modelos de aprendizagem profunda.

\section{Referências}

Ab'Sáber, A. N. (1997). O caráter diferencial das diretrizes para uso e preservação da natureza, a nível regional no brasil.

Braga, A. L. and Figueiredo, G. C., S. F. S. V. P. (2006). Comparação entre as classificações híbrida e supervisionada no mapeamento do uso do solo usando imagens de alta resolução. Anais do Congresso Brasileiro de Cadastro Técnico Multifinalitário.

Cavalcanti L., A. M., Carvalho J., H., and Miranda dos Santos, E. (2015). A comparison on supervised machine learning classification techniques for semantic segmentation of aerial images of rain forest regions. Proceedings of the 10th International Conference on Computer Vision Theory and Applications (VISAPP-2015).

Curran, P. J. (1988). The semi-variogram in remote sensing: an introduction.

Dell' Acqua, F. and Gamba, P. (2003). Texture-based characterization of urban environments on satellite sar images. IEEE Trans. on Geoscience and Remote Sensing, 41(1):153-159. 
Du, P., Samat, A., Waske, B., Liu, S., and Li, Z. (2015). Random forest and rotation forest for fully polarized sar image classification using polarimetric and spatial features. Journal of Photogrammetry and Remote Sensing (ISPRS), pages 38-53.

Fernandes, C. d. E. (2008). Classificação de imagens de sensoriamento remoto com áreas desmatadas.

He, C., Li, S., Liao, Z., and Liao, M. (2013). Texture classification of polsar data based on sparse coding of wavelet polarization textons. IEEE Transactions on Geoscience and Remote Sensing, 51(8):4576-4590.

Jin, H., Mountrakis, G., and Stehman, S. V. (2014). Assessing integration of intensity, polarimetric scattering, interferometric coherence and spatial texture metrics in PALSAR-derived land cover classification. ISPRS Journal of Photogrammetry and Remote Sensing, 98:70-84.

Kumar, M. and Miller, D. A. (2006). Non-parametric classification strategy for remotely sensed images using both spectral and textural information. International MultiConference Signal Processing, Pattern Recognition (IASTED), pages 81-89.

Magnabosco, D., Chambers, J., Higuchi, N., Trumbore, S., Ribeiro, G. H., dos Santos, J., Negrón-Juárez, R., Reu, B., and Wirth, C. (2014). Large-scale wind disturbances promote tree diversity in a central amazon forest. PLoS ONE, 9:e103711.

MapBiomas (2015). Projeto de mapeamento anual da cobertura e uso do solo no brasil. projeto mapbiomas - coleção 3.1 da série anual de mapas de cobertura e uso de solo do brasil. disponível em: http://mapbiomas.org. acesso em 2019.

Olofsson, P., Foody, G., Herold, M., Stehman, S., Woodcock, C., and Wulder, M. (2014). Good practices for estimating area and assessing accuracy of land change. Remote Sensing of Environment, page 42-57.

Pla, F.; Carmona, P. L. S. J. M. (2013). One-class classification techniques in image recognition problems. Information Optics (WIO), pages 1-3.

Qi, Z., Yeh, A. G.-O., Li, X., and Lin, Z. (2012). A novel algorithm for land use and land cover classification using RADARSAT-2 polarimetric SAR data. Remote Sensing of Environment, 118:21-39.

Russell, S. and Norvig, P. (2003). Artificial Intelligence - A Modern Approach. Prentice Hall, 2th edition.

Tan, P.-N., Steinbach, M., and Kumar, V. (2009). Introdução ao Data Mining. Ciência Moderna, 1 th edition.

Uhlmann, S. and Kiranyaz, S. (2014). Classification of dual- and single polarized SAR images by incorporating visual features. ISPRS Journal of Photogrammetry and Remote Sensing, 90:10-22.

Wang, X. e. a. (2013). A one-class classification by spatial-contextual for remotely sensed image. eoscience and Remote Sensing Symposium (IGARSS), page 437-440.

Witten, I. H. and Frank (2005). Data Mining - Practical Machine Learning Tools and Techniques. McGraw- Hill. 